\title{
Effects of Atorvastatin on Vitamin D Levels in Patients With Acute Ischemic Heart Disease
}

\author{
José L. Pérez-Castrillón, MD ${ }^{\mathrm{a} *}$, Gemma Vega, MD ${ }^{\mathrm{a}}$, Laura Abad, MD ${ }^{\mathrm{a}}$, Alberto Sanz, MD ${ }^{\mathrm{b}}$, \\ José Chaves, $\mathrm{MD}^{\mathrm{c}}$, Gonzalo Hernandez, $\mathrm{MD}^{\mathrm{c}}$, and Antonio Dueñas, $\mathrm{MD}^{\mathrm{a}}$
}

\begin{abstract}
Vitamin D deficiency is a risk factor for osteoporosis and other chronic diseases, including type 1 diabetes, hypertension, metabolic syndrome, and ischemic heart disease. Cholesterol and vitamin D share the 7-dehydrocolesterol metabolic pathway. This study evaluated the possible effect of atorvastatin on vitamin $D$ levels in patients with acute ischemic heart disease. Eighty-three patients (52 men and 31 women) with an acute coronary syndrome (75 with acute myocardial infarction and 8 with unstable angina) were included. After diagnosis, patients received atorvastatin as secondary prevention. Serum vitamin D was measured by high-performance liquid chromatography at baseline and at 12 months. Atorvastatin treatment produced a statistically significant decrease in cholesterol and triglyceride levels and an increase in vitamin D levels $(41 \pm 19$ vs $47 \pm 19 \mathrm{nmol} / \mathrm{L}, \mathrm{p}=$ 0.003). Vitamin D deficiency was decreased by $75 \%$ to $57 \%$ at 12 months. In conclusion, atorvastatin increases vitamin $\mathrm{D}$ levels. This increase could explain some of the beneficial effects of atorvastatin at the cardiovascular level that are unrelated to cholesterol levels. (C) 2007 Elsevier Inc. All rights reserved. (Am J Cardiol 2007;99:903-905)
\end{abstract}

Vitamin D deficiency is a risk factor for osteoporosis and other chronic diseases, including type 1 diabetes, hypertension, metabolic syndrome, and ischemic heart disease. ${ }^{1} \mathrm{Vi}$ tamin $\mathrm{D}$ is synthesized in the skin by ultraviolet radiation that acts on 7-dehydrocolesterol, which is hydroxylated into carbon- 25 by 25 -hydroxyvitamin $\mathrm{D}-1 \alpha$ hydroxylase or CYP27B1, an enzyme located in the mitochondria of the hepatocyte. The resulting metabolite, 25-hydroxyvitamin D, is the best way to measure individual vitamin D levels. ${ }^{2}$ Cholesterol and vitamin D share the 7-dehydrocolesterol metabolic pathway. ${ }^{3}$ Statins have beneficial effects on morbidity and mortality of patients with acute ischemic heart disease; these may be mediated by vitamin $\mathrm{D}$. These patients have a greater prevalence of vitamin D deficiency and are often treated with statins as secondary prevention. ${ }^{1}$ This led us to study the possible effect of atorvastatin on vitamin $D$ levels in this group of patients. Increased levels of vitamin D could explain some of the beneficial effects of atorvastatin at the cardiovascular and bone metabolism levels that are unrelated to cholesterol levels.

\section{Methods and Results}

Patients hospitalized for an acute coronary syndrome, defined as high-risk unstable angina, non-ST-elevated myocardial infarction, or ST-elevated myocardial infarction, were eligible for inclusion. Eighty-three patients (52 men and 31 women) with an acute coronary syndrome (75 with

\footnotetext{
${ }^{a}$ Internal Medicine Department, Río Hortega University Hospital, Faculty of Medicine of Valladolid, and 'Pediatric Laboratory, PediatricsIBGM Department, Faculty of Medicine of Valladolid, Valladolid, and ${ }^{c}$ Pfizer, Madrid, Spain. Manuscript received September 20, 2006; revised manuscript received and accepted November 7, 2006.

This study was supported by Pfizer, Madrid, Spain.

*Corresponding author: Tel: 34-983-420-400; fax: 34-983-331-566.

E-mail address: castrv@terra.es (J.L. Pérez-Castrillón).
}

Table 1

Effect of atorvastatin on vitamin D and other laboratory data

\begin{tabular}{lccc}
\hline Variable & Baseline & $12 \mathrm{mos}$ & $\begin{array}{c}\mathrm{p} \\
\text { Value }\end{array}$ \\
\hline Total cholesterol (mg/dl) & $182 \pm 48$ & $161 \pm 33$ & 0.0001 \\
HDL cholesterol (mg/dl) & $39 \pm 12$ & $49 \pm 11$ & 0.0001 \\
LDL cholesterol (mg/dl) & $114 \pm 41$ & $90 \pm 31$ & 0.0001 \\
Triglycerides (mg/dl) & $152 \pm 91$ & $117 \pm 66$ & 0.003 \\
Calcium (mg/dl) & $9.5 \pm 0.6$ & $9.6 \pm 0.5$ & $\mathrm{NS}$ \\
Phosphorus (mg/dl) & $3.6 \pm 0.6$ & $3.4 \pm 0.6$ & $\mathrm{NS}$ \\
25-Hydroxycholecalciferol (nmol/L) & $41 \pm 19$ & $47 \pm 19$ & 0.003 \\
\hline
\end{tabular}

$\mathrm{LDL}=$ low-density lipoprotein.

acute myocardial infarction and 8 with unstable angina) were included. Patients were recruited at hospital admission. Exclusion criteria were alcoholism, neoplasia, hyperor hypocalcemia, and treatment with phosphocalcium metabolism-modifying drugs. After diagnosis, patients received atorvastatin as secondary prevention. Low (10 to 20 $\mathrm{mg}$ ) and high (40 to $80 \mathrm{mg}$ ) doses were used according to baseline levels of cholesterol and triglycerides and index of vascular risk. Only patients completing follow-up were evaluated. A control group of 73 hypertensive patients (38 men and 35 women) not receiving treatment with statins was included.

Blood samples were obtained after 8 or 9 hours of fasting. Total calcium, phosphorus, total cholesterol, and triglycerides were measured using a Hitachi 917 autoanalyzer (Hitachi, Tokyo, Japan). Levels of 25-hydroxyvitamin D were determined by high-performance liquid chromatography with an intra-assay variation coefficient of $12 \%$. Laboratory measurements were obtained at baseline and at 12 months. Vitamin D deficiency was defined as $\leq 50 \mathrm{nmol} / \mathrm{L} .^{3}$

A descriptive statistical analysis was made, including measurements of central tendency and scattering for quantitative variables. Student's $t$ test was used to compare 


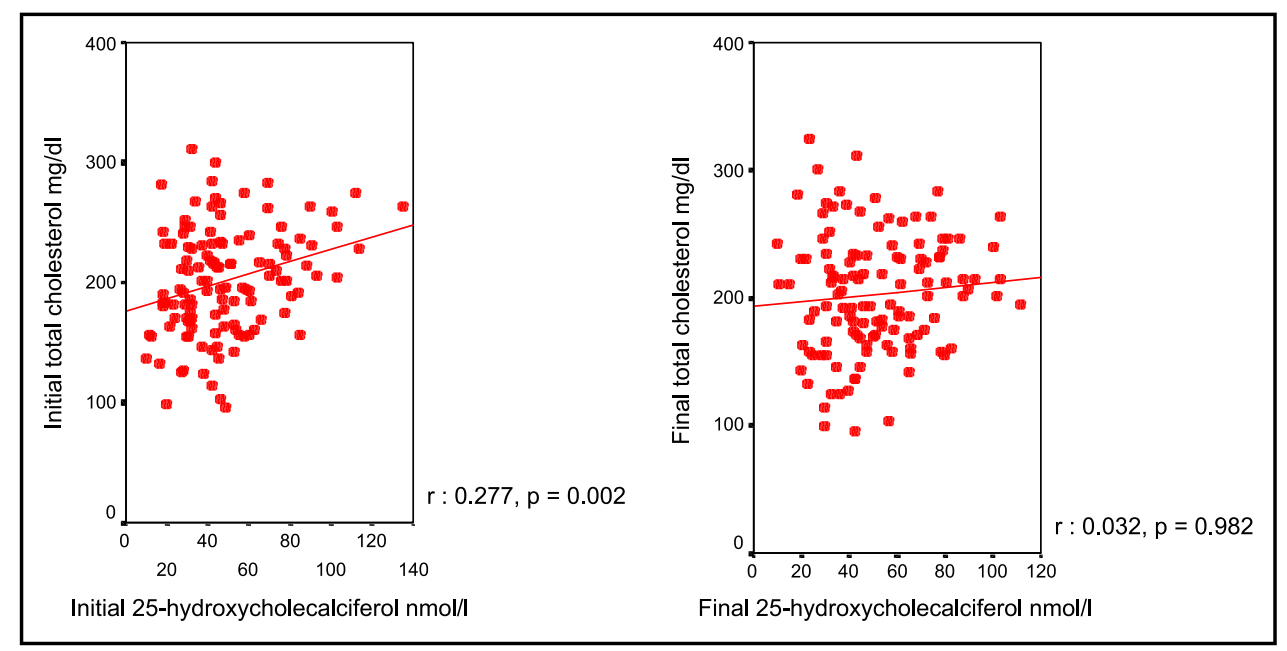

Figure 1. Relation between baseline and final levels of total cholesterol and 25-hydroxycholescalciferol.

variables and Pearson's correlation coefficient for relations between quantitative variables. Results are expressed as mean $\pm \mathrm{SD}$; statistical significance was established as $\mathrm{p}=$ 0.05 .The study was approved by the hospital clinical research committee, and all patients gave written informed consent to participate in the study.

Eighty-three patients with acute coronary syndrome and a mean age of $61 \pm 10$ years were recruited. Of these, $90 \%$ had acute myocardial infarction and $10 \%$ had unstable angina. Twenty percent of the patients were hypertensive and $13 \%$ diabetic. In addition to statins, $28 \%$ received treatment with angiotensin-converting enzyme inhibitors, $57 \%$ with $\beta$ blockers, $20 \%$ with nitrates, $2 \%$ with angiotensin antagonists, and $1 \%$ with thiazides. Fifteen percent had been previously treated with cholesterol-lowering drugs (fibrates, pravastatin). As presented in Table 1, atorvastatin treatment produced a statistically significant decrease in total cholesterol, low-density lipoprotein cholesterol, and triglyceride levels and an increase in high-density lipoprotein (HDL) cholesterol and vitamin D levels $(41 \pm 19$ vs $47 \pm 19$ $\mathrm{nmol} / \mathrm{L}, \mathrm{p}=0.003)$. Seventy-five percent of patients had vitamin $\mathrm{D}$ deficiency $(\leq 50 \mathrm{nmol} / \mathrm{L})$ at baseline, which was decreased by $75 \%$ to $57 \%$ at 12 months. ${ }^{3}$ Results did not differ according to gender or dose of atorvastatin used. A statistically significant correlation was observed between baseline levels of 25-hydroxycholecalciferol and total cholesterol $(r=0.273, p=0.002$; Figure 1$)$ and HDL cholesterol $(r=0.387, p=0.005)$. There was no relation between final levels of 25-hydroxycholecalciferol and total cholesterol $(\mathrm{r}=0.032, \mathrm{p}=0.982$; Figure 1$)$ and HDL cholesterol $(\mathrm{r}=0.078, \mathrm{p}=0.587)$. No relation between 25-hydroxycholecalciferol and low-density lipoprotein cholesterol at basal $(r=0.105, p=0.464)$ or final $(r=0.153, p=0.287)$ level was observed. Patients in the control group were an average of $62 \pm 9$ years of age and were treated with angiotensin-converting enzyme inhibitors. They had initial levels $55 \pm 26 \mathrm{nmol} / \mathrm{L}$ of vitamin $\mathrm{D}$, which were not modified during the year of follow-up $(57 \pm 25 \mathrm{nmol} / \mathrm{L}$, $\mathrm{p}$ $=0.543$ ).

\section{Discussion}

These results show that atorvastatin significantly increased vitamin D levels, thus decreasing the percentage of patients with vitamin $\mathrm{D}$ deficiency, a previously unrecorded finding. Comparable findings come from 2 small studies that included 18 patients with familial hypercholesterolemia, which showed that lovastatin and simvastatin treatments resulted in increased plasma levels of vitamin D. ${ }^{4,5}$ Grimes $^{6}$ analyzed analogies between vitamin D and statins and suggested that part of the benefits obtained by these drugs could be mediated by vitamin D. Our results support this hypothesis.

The possible interest of vitamin D in cardiovascular disease is because patients with terminal renal disease and low levels of vitamin D have a cardiovascular mortality 10 to 20 times greater than that of the general population. ${ }^{1}$ Vitamin D deficiency may increase the risk of cardiovascular disease through 3 mechanisms. First, in vitro studies have shown that vitamin D causes an imbalance between anti-inflammatory and proinflammatory cytokines, thus decreasing the activity of nuclear factor- $\kappa \mathrm{B}$, increasing the production of interleukin-10, and decreasing levels of interleukin-6, interleukin-1, interferon- $\gamma$, and tumor necrosis factor- $\alpha .{ }^{7}$ Second, myocardial cells of the vascular endothelium have receptors for vitamin $\mathrm{D}^{1}$ whose activation inhibits cell proliferation. Local production of calcitriol, the active metabolite of vitamin $\mathrm{D}$, depends on circulating levels of 25-hydroxycholecalciferol, which would be increased by atorvastatin treatment. Third, the association between vitamin deficit and hypertension is mediated by activation of the renin-angiotensin-aldosterone system. High levels of calcitriol decrease plasma renin activity, producing a decrease in angiotensin II levels. ${ }^{8}$ This modulation of the renin-angiotensin-aldosterone system, in addition to decreasing blood pressure, decreases inflammation at the level of the vascular endothelium, thus limiting progression of atherosclerosis. ${ }^{9}$

The mechanism by which atorvastatin increases vitamin D levels is related to inhibition of 3-hydroxy-3 methylglutaryl coenzyme A (HMG-CoA) reductase. Cholesterol is 
synthesized from 7-dehydrocholesterol, which is also a precursor of vitamin $\mathrm{D}_{3}$. For this reason, we initially observed a statistically significant relation between total cholesterol and vitamin D. HMG-CoA enzyme reductase is key to the synthesis of cholesterol, whereas ultraviolet radiation causes the formation of 25-hydroxyvitamin D. Inhibition of the enzyme may increase levels of 7-dehydrocholesterol and increase the synthesis of 25-hydroxycholecalciferol, thereby increasing vitamin D levels, ${ }^{10}$ although we observed no relation between lower cholesterol and increased vitamin $\mathrm{D}$. In addition, 25-hydroxyvitamin $\mathrm{D}$ has been shown to inhibit HMG-CoA enzyme reductase activity in in vitro studies. ${ }^{11}$ A greater concentration of vitamin D could increase enzymatic inhibition, acting in synergy with the statin in decreasing total cholesterol. Future studies evaluating the effect of atorvastatin on bone mineral density and markers of bone remodeling in patients with an increased risk of osteoporosis will help to clarify these relations.

1. Zittermann A. Vitamin D and disease prevention with special reference to cardiovascular disease. Prog Biophys Mol Biol 2006; 92:39-48.

2. Lips P. Vitamin D physiology. Prog Biophys Mol Biol 2006;92:4-8.
3. Hollick MF. Resurrection of vitamin D deficiency and rickets. J Clin Invest 2006;116:2062-2072.

4. Wilczek H, Sobra J, Justova V, Ceska R, Juzova Z, Prochazkova R, Kvasilova M, Pacovsky V. Iatropathogenic effect of mevacor on vitamin D metabolism. Cas Lek Cesk 1989;128:1254-1256.

5. Wilzcek H, Sobra J, Ceska R, Justota V, Juzova Z, Prochazkova R, Kvasilova M. Monitoring plasma levels of vitamin D metabolites in simvastatin therapy in patients with familial hypercholesterolemia. Cas Lek Cesk 1994;133:727-729.

6. Grimes DS. Are statins analogues of vitamin D?. Lancet 2006;368: 83-85.

7. Zittermann A, Scheleithoff SS, Koerfer R. Vitamin D insufficiency in congestive heart failure: why and what to do about it? Heart Fail Rev 2006; 11:25-33.

8. Li YC. Vitamin D regulation of the renin-angiotensin system. $J$ Cell Biochem 2003;88:327-331.

9. Ferrario CM, Strawn WB. Role of the renin-angiotensin-aldosterone system and proinflammatory mediators in cardiovascular disease. Am J Cardiol 2006;98:121-128.

10. Guryev O, Carvalho RA, Usanov S, Gilep A, Estabrook RW. A pathway for the metabolism of vitamin D3: unique hydroxylated metabolites formed during catalysis with cytochrome P450scc (CYP11A1). Proc Natl Acad Sci USA 2003;100:14754-14759.

11. Gupta AK, Sexton RC, Rudney H. Effects of vitamin D3 derivatives on cholesterol synthesis and HMG-CoA reductase activity in cultured cells. J Lipid Res 1989;30:379-386. 